\title{
OCULAR PAIN: A CASUALTY STUDY. THE SPECTRUM AND PREVALENCE OF PAIN IN ACUTE EYE DISEASE
}

\author{
C. J. ROBERTS, J. D. A. MacLEOD and A. R. ELKINGTON \\ Southampton
}

\section{SUMMARY}

This study examined the prevalence and severity of ocular pain in eye casualty patients. All new patients presenting over a 1 month period were invited to indicate their pain level using a visual analogue scale. The results for 352 patients were analysed, and median pain levels calculated for 29 common diagnoses. Of those responding, 94\% (= 47\% of all new patients) had ocular pain at presentation. While many results were predictable, some diagnoses were associated with higher pain scores than expected. Junior ophthalmologists also were asked to indicate their perceptions of pain severity for the 29 diagnoses using the same visual analogue scale.

Pain is a common feature of many acute eye diseases. ${ }^{1,2}$ Unlike other ocular symptoms, such as reduced visual acuity, it is entirely subjective and may be difficult to assess in clinical practice. The visual analogue scale has been shown to be a reproducible tool for quantifying pain ${ }^{3,4}$ and has been used in ophthalmology to study post-operative analgesia, ${ }^{5-8}$ as well as the treatment of specific conditions such as corneal abrasion. ${ }^{9}$

However, there is still little information available about the prevalence and severity of pain in the large group of ophthalmic patients who attend eye casualty with acute eye symptoms.

\section{MATERIALS AND METHODS}

All new patients attending a busy eye casualty over a 1 month period (July 1994) were given a proforma on which they were invited to indicate current pain scores for each eye on a $10 \mathrm{~cm}$ horizontal visual analogue score ( 0 , no pain; 10 , maximum pain). New patients were defined as all attending patients except those under treatment by the Eye Unit for their

Correspondence to: Miss C. J. Roberts, FRCOphth, Eye Department, Queen Alexandra Hospital, Portsmouth PO6 3LY, UK. current problem. No patients included had attended casualty in the preceding 4 months. Patients who were unable to understand and complete the proforma were excluded from the study. Following examination and treatment, the casualty staff then recorded the diagnosis and duration of the symptoms on the proforma.

Nine ophthalmologists working regularly in the casualty department were asked to record their perceptions of pain severity for the 29 diagnoses, using the visual analogue scale. The doctors were unaware of the results of the main study.

\section{RESULTS}

During the study period 757 patients presented to the department with new eye problems. Fifty-eight of these were children who were unable to participate. Of the remainder, $381(55 \%)$ returned proformas, and $352(50 \%)$ of these contained sufficient data for analysis.

Forty-three (12\%) of the 352 patients had bilateral pain, $288(82 \%)$ had unilateral pain, and $21(6 \%)$ recorded no pain in either eye. In 9 patients, no abnormal signs were found; 6 of these had recorded symptoms of pain. Median patient pain scores and ophthalmologists' estimated pain level for each diagnosis are shown in Fig. 1. No correlation was found between the pain score and the time elapsed between onset of symptoms and attendance. The estimation of pain levels for the various diagnoses by the ophthalmologists was within \pm 0.5 of the patient's pain score in 9 of the 29 diagnoses $(31 \%)$, lower in 7 conditions $(24 \%)$, but an overestimate of pain levels in 13 diagnoses $(45 \%)$.

\section{DISCUSSION}

This study suggests. that pain is a feature in at least $47 \%$ of new presentations to an eye casualty department. Ninety-four per cent of those completing the proforma recorded this symptom. It is 
Diagnosis

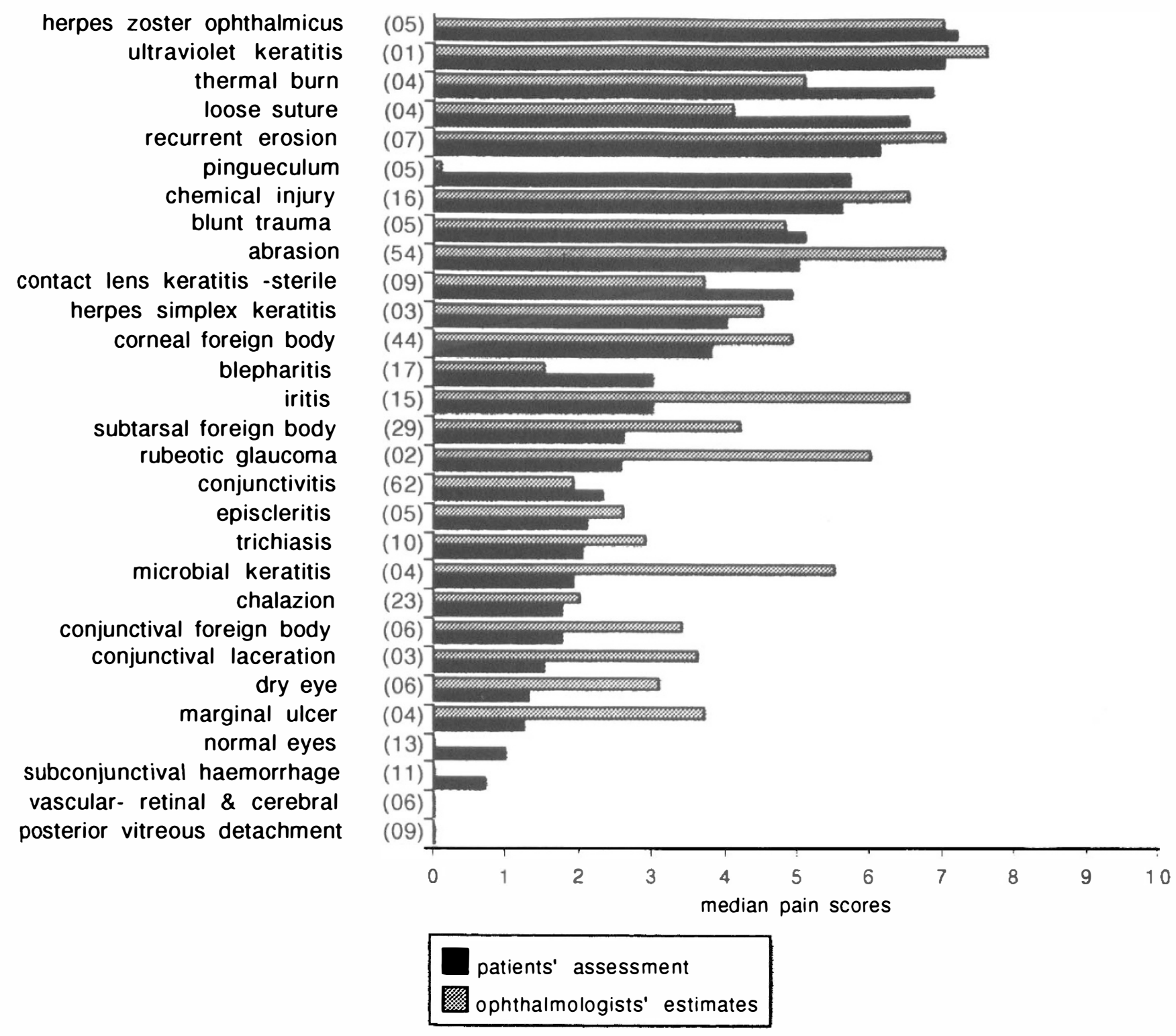

Fig. 1. Median pain scores for each diagnosis as indicated by patients (black bars) and by ophthalmologists (hatched bars). Numbers of patients presenting with each condition are shown in parentheses

possible that a large proportion of those who did not return proformas had no pain. While the number of non-respondents is a limitation of this study, we did not feel it was appropriate, in a casualty department, to take further measures to encourage participation.

The pain scores for most specific diagnoses were as expected: thus herpes zoster ophthalmicus had the highest pain score (median 7.2), and other high scores were predominantly for corneal conditions, including 'arc eye'. This is unsurprising given the uniquely dense nociceptive innervation of the cornea. ${ }^{2}$ Amongst other conditions frequently described as very painful is acute glaucoma, but this did not present during the study period. In contrast, con- junctival disorders were grouped towards the lower end of the scale, while non-inflammatory posterior segment disorders had median scores of 0 . The high scores elicited for some conditions are more surprising - for example loose corneal sutures (score of 6.5) and inflamed pingueculum (5.7) - and it is instructive that blepharitis was given the same median score as iritis. Clearly, patient discomfort must be given due weight in the management and prevention of some apparently minor eye conditions.

We predicted that more painful conditions would present sooner, but found no correlation. While blepharitis had the longest median duration of symptoms (14 days) and subtarsal foreign body one 
of the shortest ( 2 hours), median pain scores were similar for each of these conditions.

The assessment of pain levels by ophthalmologists working in casualty correlated well with patients' pain scores in the more painful conditions whilst in less painful conditions over- rather than underassessment was the norm. Overall in this study only $24 \%$ of diagnoses were considered less painful by the ophthalmologists than by the affected patients (blepharitis, loose suture, pingueculum, subconjunctival haemorrhage, normal eyes, sterile contact lens keratitis and thermal burn). Subjective pain scores reflect both severity of nociceptive stimuli and the psychological response to these stimuli. It seems likely that patient anxiety is a significant factor in the pain caused by some of these conditions and so explanation and reassurance are a vital part of pain management.

Ocular pain appears to be a common presenting symptom in an eye casualty department. This study has attempted to quantify the pain caused by common ocular conditions and to compare pain levels attributed by ophthalmologists to common diagnoses with the patients' own assessments of their pain.
Key words: Pain, Eye, Visual analogue scale.

\section{REFERENCES}

1. Rosenblatt MA, Sakol PJ. Ocular and periocular pain. Otolaryngol Clin North Am 1989;22:1173-203.

2. Tanelian DL, Brunson DB. Anatomy and physiology of pain with special reference to ophthalmology. Invest Ophthalmol Vis Sci 1994;35:759-62.

3. Revill SI, Robinson JO, Rosen M, Hogg MIJ. The reliability of a linear analogue for evaluating pain. Anaesthesia 1976;31:1191-8.

4. Chapman CR, Casey KL, Dubner R, Foley KM, Gracely RH, Reading AE. Pain measurement: an overview. Pain 1985;22:1-31.

5. Koay P. Ophthalmic pain following cataract surgery: a comparison between local and general anaesthesia. Br J Ophthalmol 1992;76:225-7.

6. Stevens JD. A new local anaesthesia technique for cataract extraction by one quadrant sub-Tenon's infiltration. Br J Ophthalmol 1992;76:670-4.

7. El-Kasaby HT, Habib NE, Marczac AM. Subconjunctival bupivacaine in strabismus surgery. Eye 1993;7:346-9.

8. Morrison NA, Repka MX. Ketorolac versus acetaminophen or ibuprofen in controlling postoperative pain in patients with strabismus. Ophthalmology 1994;101: 915-8.

9. Kirkpatrick JNP, Hoh HB, Cook SD. No eye pad for corneal abrasion. Eye 1994;7:468-71. 